\title{
YTHDF3 as a new player in hematopoietic stem cell regulation
}

Xinjian $\mathrm{MaO}^{1}$ and Linheng $\mathrm{Li}^{1,2}$

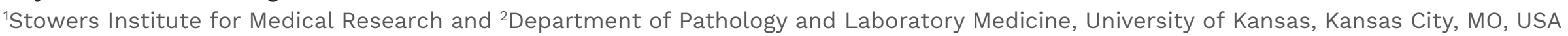
E-mail: LIL@stowers.org

https://doi.org/10.3324/haematol.2021.280467

Hematopoietic stem cells (HSC) are characterized by selfrenewal and multipotent differentiation potential, and are required to maintain the hematopoietic system throughout life. Since HSC transplantation is a life-saving treatment for various hematopoietic disorders, dissecting the mechanisms underlying how intrinsic programs and extrinsic niche signals are orchestrated to regulate HSC in vivo would enable development of new methods for ex vivo HSC expansion, and would potentially benefit clinical treatment for blood disorders.

N6-methyladenosine $\left(\mathrm{m}^{6} \mathrm{~A}\right)$ is the most prevalent messenger RNA ( $m R N A$ ) modification in mammals. ${ }^{1}$ The proteins involved in the deposition, removal, and execution of the $\mathrm{m}^{6} \mathrm{~A}$ modification are referred to as $\mathrm{m}^{6} \mathrm{~A}$ 'writers', 'erasers' and 'readers', respectively. ${ }^{2}$ Over the last decade, the $\mathrm{m}^{6} \mathrm{~A}$ modification has been demonstrated to play essential and broad roles in the regulation of RNA stability and translation in various types of cells, although the detailed mechanisms require further exploration and seem to be cell- and context-dependent. ${ }^{2,3}$ In recent years, several studies have revealed that deficiency of the $\mathrm{m}^{6} \mathrm{~A}$ writer Mettl3 or Mettl14 impairs self-renewal and differentiation capacity of HSC. . $^{4-6}$ In contrast, two groups independently discovered that loss of Ythdf2 promotes HSC expansion and regeneration. ${ }^{7,8}$ Given that the $\mathrm{m}^{6} \mathrm{~A}$ readers are the primary component responsible for exerting the function of $\mathrm{m}^{6} \mathrm{~A}$-containing RNA deposited by METTL3, an important question is: which readers are responsible for the characteristics of $\mathrm{Mett}^{-/-} \mathrm{HSC}$ ?

As described in their paper in this issue of Haematologica, to test the hypothesis that other readers in the cytoplasm may be responsible for the hematopoietic phenotype in Mett $13^{-/-}$mice, Zhang et al. first investigated the hematopoietic system in $Y$ thdf1 ${ }^{-/-}$and $Y$ thdf $3^{-/-}$mice. ${ }^{9}$ Both the frequency and the absolute number of HSC in $\mathrm{Ythdf}^{-/-}$mice, unlike in $\mathrm{Ythdf1}^{-1-}$ mice, were significantly increased when compared with those in wild-type mice. Of note, the magnitude of the increase in $\mathrm{HSC}$ in $\mathrm{Ythdf3}^{-/-}$mice was much smaller than that in Mettl $3^{-/-}$mice. No other significant differences in hematopoietic cells were found between $y_{\text {thdf }}{ }^{-/-}$and the littermate mice. In contrast, bone marrow cellularity and hematopoietic cells declined markedly in Mett $3^{-/-}$mice. Consistent with the severely impaired reconstitution ability of $\mathrm{Mettl}^{-/-} \mathrm{HSC}$, the $\mathrm{Ythdf3}^{-/-} \mathrm{HSC}$ exhibited poor reconstitution capacity with similar multilineage differentiation, comparable homing capacity, higher sensitivity to replication stress, and diminished protein synthesis.

Given that $Y$ thdf $^{-/-}$HSC resemble Mett $3^{-/-}$HSC to some extent, and that targets of $Y$ thdf 3 and $Y$ thdf 2 overlap partially, Zhang et al. reasoned that $Y$ thdf3 may mediate HSC via reversely regulating the targets of Ythdf2. Among six mRNA previously identified to be increased in $\mathrm{Ythdf2}^{-/-} \mathrm{HSC},{ }^{8}$ only Cond1 was decreased in Ythdf $3^{-/-}$HSC. Based on these findings, the next question to be explored in the future is: how do YTHDF2 and YHTDF3 orchestrate the regulation of Ccnd1 expression and in what context do YTHDF2 and YTHDF3 each dominate such regulation? Knockdown assays confirmed that the protein level of $C \mathrm{cnd1}$, rather than the mRNA level, decreased significantly in both Ythdf3 shRNA-carrying and Mettl3 shRNA-carrying HSC. This result is congruent with the role of the $\mathrm{m}^{6} \mathrm{~A}$ modification in regulating gene expression at a post-transcriptional level. The 5 ' untranslated region of Ccnd1 in the -180 to -184 region was identified as the major $\mathrm{m}^{6} \mathrm{~A}$ motif recognized by YTHDF3 and METTL3. YTHDF3 cooperated with PABPC1 and elF4G2 to promote the translation of Ccnd1. Furthermore, knockdown of Ccnd1 recapitulated the compromised reconstitution capacity of Ythdf3 ${ }^{-/-}$HSC. On the other hand, forced expression of Ccnd1 completely rescued the reconstitution potential of $Y$ thdf3 shRNA-carrying HSC, but not of Mettl3 shRNA-carrying HSC. Myc has previously been reported to be an important target gene of Mettl3, and enforced expression of Myc rescued the lineage differentiation bias of $M e t t l 3^{-/-}$HSC. To investigate the role of $M y c$ in reconstitution potential, Zhang et al. overexpressed Myc in Mettl $3^{-/-}$HSC and evaluated the engraftment capacity of these cells. The result showed that forced expression of Myc failed to rescue the reconstitution ability of $\mathrm{Mettl}^{-3^{--}} \mathrm{HSC}$, although it consistently rescued the lineage differentiation bias of Mettl $3^{-/-}$HSC. These data indicate that, besides $Y$ thdf3 and Myc, other unknown players are responsible for the characteristics of $\mathrm{Mett} \mathrm{3}^{-/-} \mathrm{HSC}$. Could the other $\mathrm{m}^{6} \mathrm{~A}$ readers (e.g., YTHDC1 and YTHDC2) be the missing link?

The $\mathrm{m}^{6} \mathrm{~A}$ modification has recently been discovered to play a critical role in the regulation of HSC, although the mechanisms are still not fully understood. Zhang et al. were able to establish that the Mettl3-Ccnd1-Ythdf3 axis regulates the characteristics of HSC. Their study uncovers the missing piece of the puzzle by revealing that the $\mathrm{m}^{6} \mathrm{~A}$ reader YTHDF3 is partially responsible for Mettl 3 deficiency in HSC, broadening the knowledge of how RNA $\mathrm{m}^{6} \mathrm{~A}$ components el- 


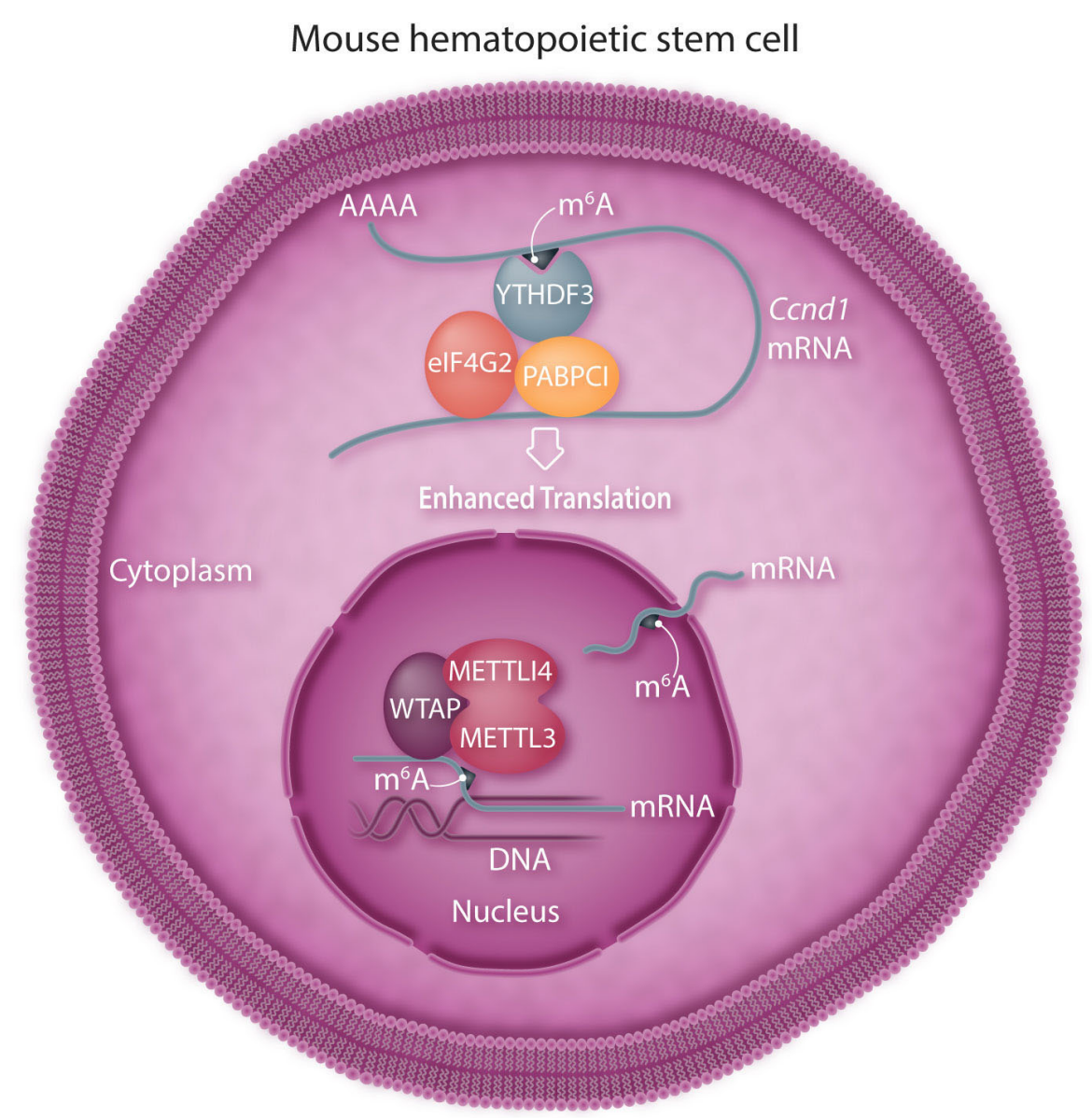

Figure 1. Proposed model of the Mettl3 $\rightarrow$ RNA m $^{6} \mathrm{~A} \rightarrow$ Ccnd1 $\rightarrow$ Ythdf3 axis regulating the reconstitution capacity of mouse hematopoietic stem cells. METTL3, along with other components including METTL14 and WTAP, form a complex and deposit $\mathrm{m}^{6} \mathrm{~A}$ to Ccnd1 mRNA in the nucleus. The $\mathrm{m}^{6} \mathrm{~A}$-containing Cond1 mRNA then travels from the nucleus to the cytoplasm for translation. YTHDF3 specifically recognizes the $\mathrm{m}^{6} \mathrm{~A}$ motif, mainly at the 5' UTR of Ccnd1 mRNA in the region from -180 to -184 , and increases the expression of $\mathrm{Ccnd} 1$ by recruiting PABPC1 and elF4G2 to enhance protein synthesis. The up- regulated expression of Ccnd1 is responsible for YTHDF3-mediated reconstitution capacity of mouse hematopoietic stem cells. egantly combine to regulate HSC (Figure 1). Based on this discovery, there are several questions to be explored further in the future: (i) Besides MRNA, METTL3 has recently been discovered to influence chromatin state and transcription of mouse embryonic stem cells via YTHDC1-mediated regulation of chromosome-associated regulatory RNA. Is this regulatory mechanism also applicable to adult stem cells, for example HSC? (ii) Do the regulatory mechanisms that govern mouse HSC properties, such as self-renewal and differentiation, also regulate similar properties in human HSC? Although a study previously demonstrated the opposite role of Mettl3 in human hematopoietic stem and progenitor cells and in murine HSC, a series of experiments using purified human HSC, as opposed to human $\mathrm{CD} 34^{+}$hematopoietic stem and progenitor cells, which only contain a very small population of HSC, is warranted to answer this question. (iii) Given that Mettl3 deficiency leads to increased numbers and decreased differentiation of HSC, and that rapid differentiation of HSC in in vitro culture is a major challenge in the field, a pertinent question becomes: is it possible to inhibit METTL3 pharmacologically using small molecules to block differentiation of HSC in ex vivo cultures?

\section{Disclosures}

No conflicts of interest to disclose.

\section{Contributions}

$X M$ wrote the editorial; $L L$ supervised and edited it.

\section{References}

1. Dominissini D, Moshitch-Moshkovitz S, Schwartz S, et al. Topology of the human and mouse m6A RNA methylomes revealed by m6A-seq. Nature. 2012;485(7397):201-206.

2. Shi H, Wei J, He C. Where, when, and how: context-dependent functions of RNA methylation writers, readers, and erasers. Mol Cell. 2019;74(4):640-650.

3. Hsu PJ, Shi H, He C. Epitranscriptomic influences on development and disease. Genome Biol. 2017;18(1):197.

4. Cheng $Y$, Luo $H$, Izzo F, et al. m(6)A RNA methylation maintains hematopoietic stem cell identity and symmetric commitment. Cell Rep. 2019;28(7):1703-1716.

5. Lee $H$, Bao S, Qian Y, et al. Stage-specific requirement for Mettl3-dependent m(6)A mRNA methylation during haematopoietic stem cell differentiation. Nat Cell Biol.
2019;21(6):700-709.

6. Vu LP, Pickering BF, Cheng Y, et al. The N(6)-methyladenosine (m(6)A)-forming enzyme METTL3 controls myeloid differentiation of normal hematopoietic and leukemia cells. Nat Med. 2017;23(11):1369-1376.

7. Li Z, Qian P, Shao W, et al. Suppression of m(6)A reader Ythdf2 promotes hematopoietic stem cell expansion. Cell Res. 2018;28(9):904-917.

8. Wang $\mathrm{H}$, Zuo H, Liu J, et al. Loss of YTHDF2-mediated m(6)Adependent mRNA clearance facilitates hematopoietic stem cell regeneration. Cell Res. 2018;28(10):1035-1038.

9. Zhang X, Cong T, Wei L, et al. YTHDF3 modulates hematopoietic stem cells by recognizing RNA m(6)A modification on Ccnd1. Haematologica. 2022;107(10):2381-2394. 